American Journal of Applied Sciences 5 (11): 1494-1498, 2008

ISSN 1546-9239

(C) 2008 Science Publications

\title{
The Application of Multi-Agent Technology on Transient Stability Assessment of Iraqi Super Grid Network
}

\author{
${ }^{1}$ Afaneen A. Abood , ${ }^{2}$ Ahmed N. Abdalla and ${ }^{3}$ Shant K. Avakian \\ ${ }^{1}$ Department of Electrical and Electronics, \\ ${ }^{2}$ Center of Training and Work Shops, \\ ${ }^{3}$ Department of Computer Science, University of Technology, Baghdad, Iraq
}

\begin{abstract}
The aim of this research is improving the Iraqi control center's capabilities for monitoring and controlling the electrical network by adopting the properties of multi-agent technology to enhance power system transient stability. The idea was demonstrated by two agent's strategy, the first agent is a prediction one that will predict power system instability by a transient stability program using the PEBS method, the second strategy agent is a control agent which use the methodology of increasing power transfer through the healthy portion of network during disturbances by a load flow program using fast decoupled method. The proposed strategies are applied to a realistic power system, the Iraqi supper Grid electrical network. Results show that the proposed technique is very powerful for power system instability prediction and control.
\end{abstract}

Key words: Multi-agent, power system instability, Iraqi supper grid network

\section{INTRODUCTION}

For reliable service, a bulk electricity system must remain intact and be capable of withstanding a wide variety of disturbances. Therefore, it is essential that the system be designed and operated so that the more probable contingencies can be sustained with no loss of load (except that connected to the faulted element). So, the most adverse possible contingencies must not result in uncontrolled, widespread and cascading power interruptions ${ }^{[1]}$. In the past, when systems were smaller and less complicated informal methods of security, analysis and control, were performed by system operators based upon experience and knowledge of system. Modern power systems are quite large and more extensively interconnected making the task of security analysis and control difficult for the system operator $^{[2]}$. In case where transient stability is an issue, the conventional methods of stability analysis by a time domain iterative process are too far slow for online operation. This led researchers to explore fast direct methods to analyze transient stability of electric power systems $^{[3]}$. A direct method for transient stability analysis is defined as a method that is able to determine stability without explicitly integrating differential equations describing the post-fault system $^{[4]}$. Among different methods proposed, the standard Energy Approach has received a great deal of attention.
In this research a prediction agent is designed to predict the stability of the network under consideration during the abnormal conditions by applying the PEBS method for transient stability. The predicted values are linked to a control agent that will apply the control action to stabilize the system. This control action is carried out by increasing the generating power of the generating unit in the network and analyze the network by a load flow program then fed the new situation of power flow to the predicted agent so as to predict the stability of the network. The process will be repeated until reaching the stable situation

\section{THE MULTI-AGENT STRUCTURE}

The idea of designing the new structure was to increase the generation of the generating units in steps in the case of instability of the system after a disturbance. This increment will be added to the input data file of the load flow program (the control agent) to obtain anew power flow which is fed to the transient stability program (the predictive agent) so as to check the stability of the system. The process will continue until stable situation is reached. Figure 1 shows the proposed multi-agent's structure.

Scenario of the multi-agent technique can be illustrated as:

- Three-phase fault occurred on each generation buses 
Am. J. Applied Sci., 5 (11): 1494-1498, 2008

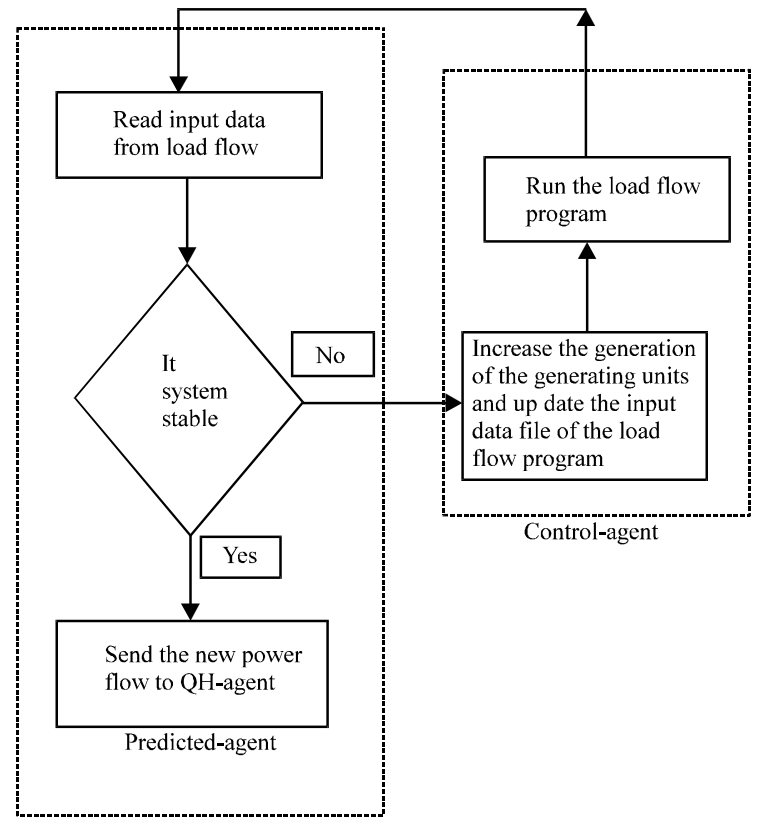

Fig. 1: The proposed multi-agent's structure

- Clearing the fault according to the calculated Critical Clearing Time (CCT)

- Observe the stability of the system

- Apply control agent to the disturbed generators

Prediction agent and control agent are linked to a measurement agent, which updates the values of the prediction and provides active power measurements for the control agent as shown in Fig. 2. This agent is represent the HQ agent in the proposed agent structure of Iraq control system ${ }^{[5]}$.

The predicted agent: The proposed prediction agent is adapted from applying a transient stability program using DML/EAF (The Direct Method of Lyapunov/ The Energy Approach Function. The interest in direct methods for transient stability analysis stems from the problem of real-time prediction of instability and control.

In PEBS Approach Function, the transient energy method can easily explained from the expression of the total energy. Most of the stability concepts can be interpreted by considering a ball sliding without friction in a bowl having a shape similar to that of the potential energy surface $\operatorname{Vpe}(\delta)$ as depicted in Fig. 3. During the fault-on period, an additional energy is injected into the system in the same way as the ball in the bowl is given energy when it is initially pushed. During the post-fault period, the total energy remains constant.

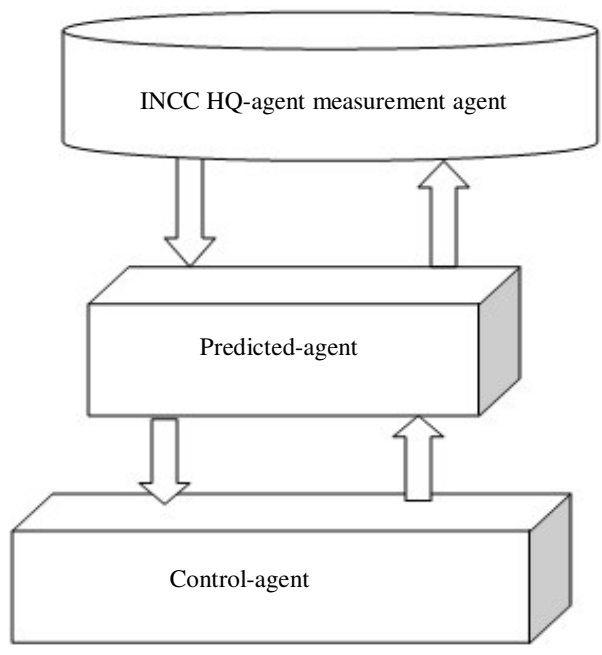

Fig. 2: Control system and multi-agents interaction.

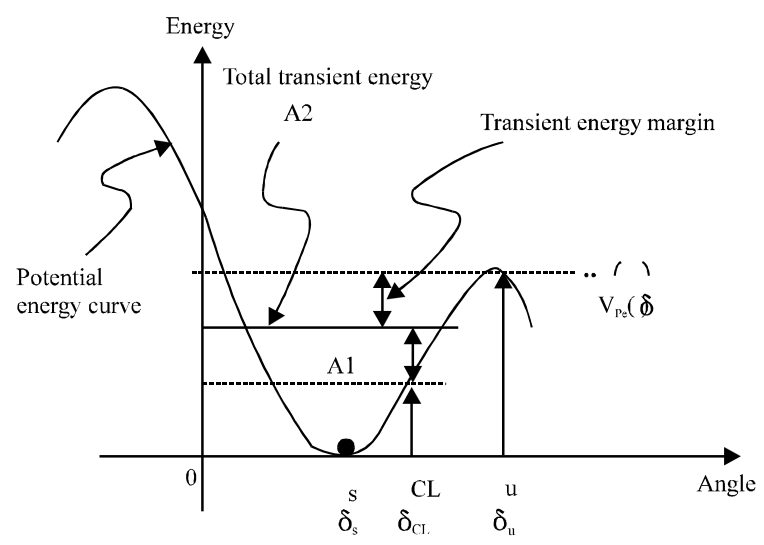

Fig. 3: TEF approach ${ }^{[6]}$

In Fig. 3, A1 represents the excess of kinetic energy injected into the system during the time period where the fault is on and A2 represents the total energy present in the system at the clearing time tc. The stability of the system is determined by the ability of the post-fault system to convert the excess kinetic energy $\mathrm{Vke}(\omega \mathrm{c})$. If the kinetic energy at clearing time exceeds the difference between the potential energy at clearing time and that at the u.e.p, the system will be unstable. Formally, we have:

$$
\mathrm{V}_{\mathrm{Ke}}\left(\omega_{\mathrm{cl}}\right)+\mathrm{V}_{\mathrm{Pe}}\left(\delta_{\mathrm{cl}}\right)<\mathrm{V}_{\mathrm{Pe}}\left(\delta_{\mathrm{u}}\right)
$$

This inequality is the mathematical way of stating the Energy Approach. The above inequality must hold not only at clearing time, but also during the post-fault time, that is: 


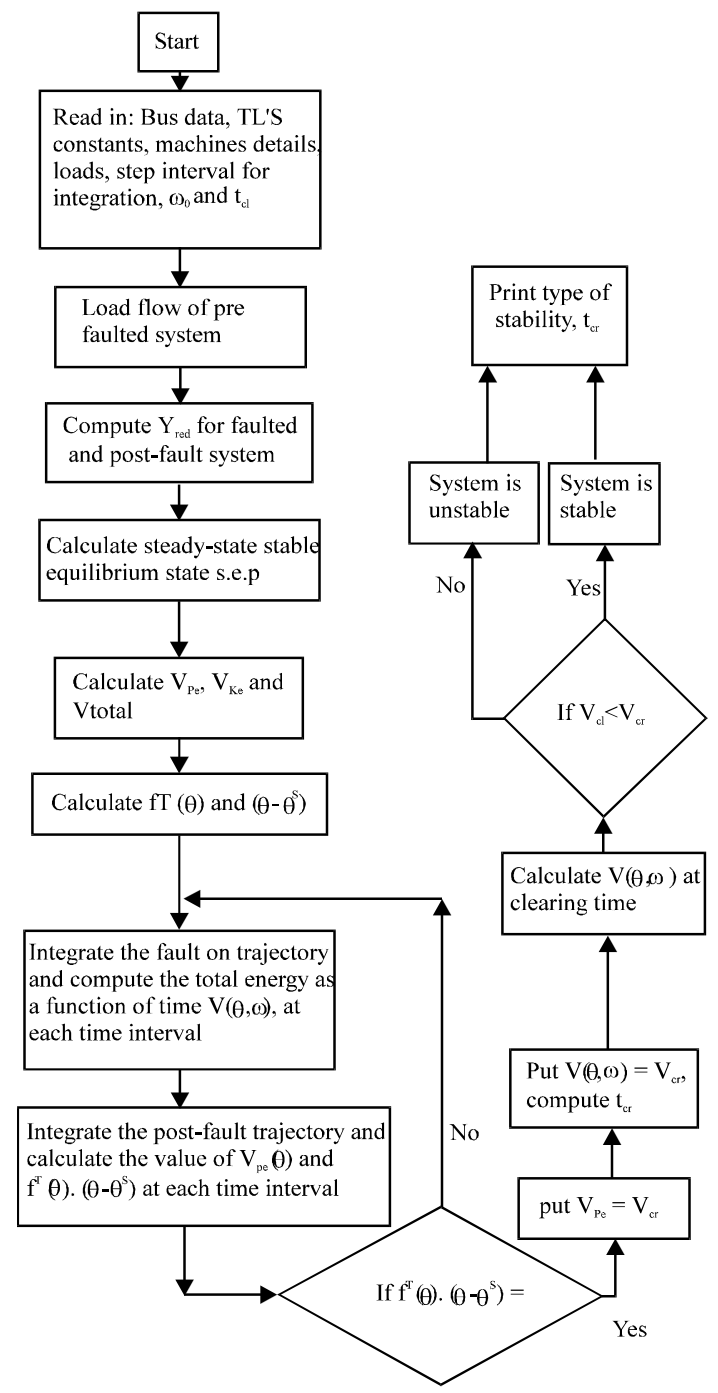

Fig. 4: The transient stability's flow chart ${ }^{[7]}$

$$
\mathrm{V}_{\mathrm{Ke}}(\omega)+\mathrm{V}_{\mathrm{Pe}}(\delta)<\mathrm{V}_{\mathrm{Pe}}\left(\delta_{\mathrm{u}}\right)
$$

According to the inequality (2), a transient stability criterion can be defined and extended to a multimachine system as follows:

Following a disturbance, the system is transiently stable if the total transient energy is less than the potential energy evaluated at the closest Unstable Equilibrium Point (UEP).

The flow chart of the transient program is shown in Fig. $4^{[6]}$.

The Control Agent: The most important mathematical operation in power system analysis is the investigation of load-flow study, which is concerned with the

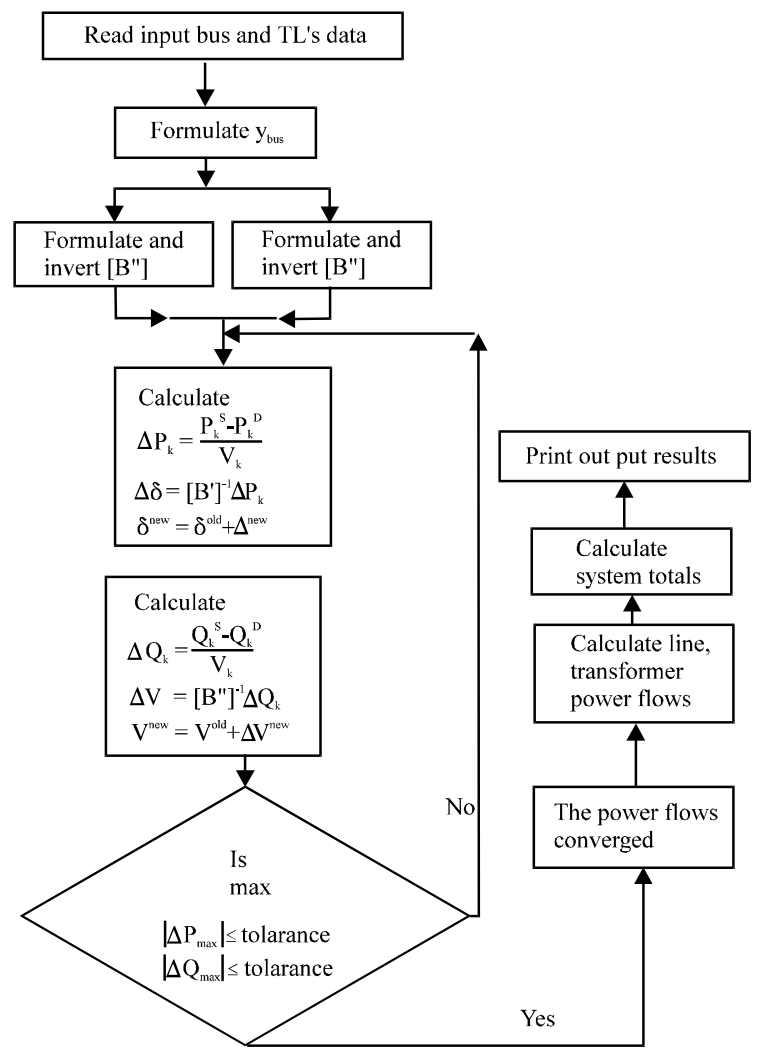

Fig. 5: Flow chart for a fast decoupled power flow $\operatorname{program}^{[7]}$

determination of state (variables voltage, current, power and power factor) at a various point of power network. Load flow studies are essential for all power system problems and it is not a simple task. No direct solution can be found to a load flow because of the nature of the equations and ever increasing complexity of power systems.

There are many powerful methods for load flow study, but the fast decoupled load flow method has been accepted in recent years by utility industry as the best approach to obtain the power flow solutions. This method is used in system planning, operational planning and operational control due to its low memory requirements, speed and very good convergence characteristics for practical problems. In this research, a load flow program was developed using the fast decoupled method as a control agent. Figure 5 shows the flow chart of the program ${ }^{[7]}$.

\section{RESULTS OF CASE STUDIE}

The network under consideration represents the Iraqi super grid network which consist of 19 bus-bar 

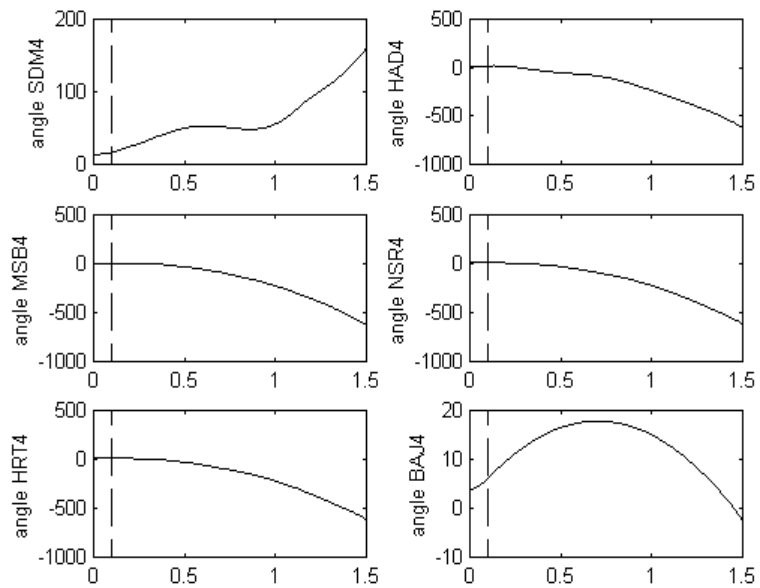

Fig. 6: $\delta$ vs. t for all generating units in case of BAJ4 outage
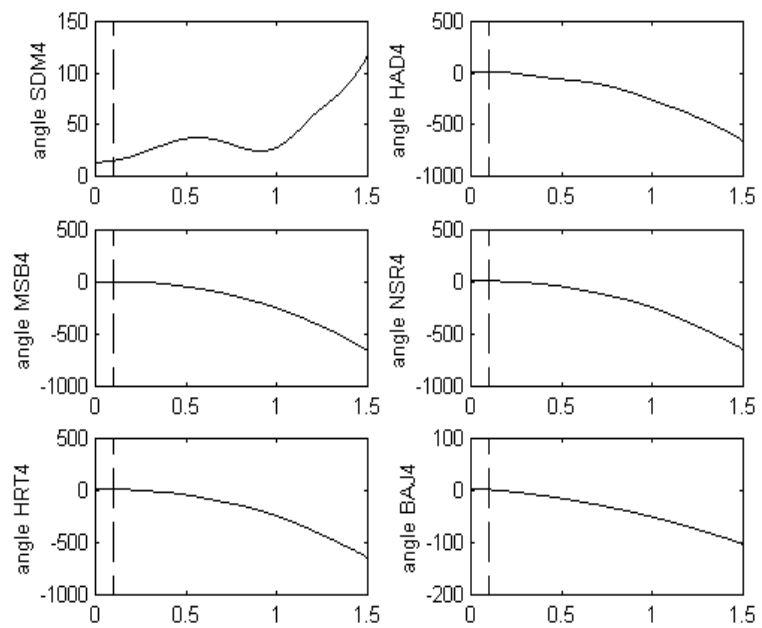

Fig. 7: $\delta$ vs. $\mathrm{t}$ for all generating units in the situation of HAD4 outage

and 30 transmission lines, the input data for the load flow and the transient stability programs represent the load and the generation of the $2 \mathrm{nd} / \mathrm{jun} . / 2000^{[8]}$.

The type of fault that was considered in this research is a three phase short circuit near the bus bar which follows the transformer of the generator. To ensure getting rid of the severe condition, the faulted line is not returned to the system. Since there are six generating bus bar in network under consideration the study tried to separate each generating unit once a time, the results shows that the stability of the system is affected only with the separation of BAJ4 and HAD4 bus bars, since the separation of those two bus bars cause the instability of SDM4 bus and so the instability of the system. Figure 6 and 7 shows the curves which
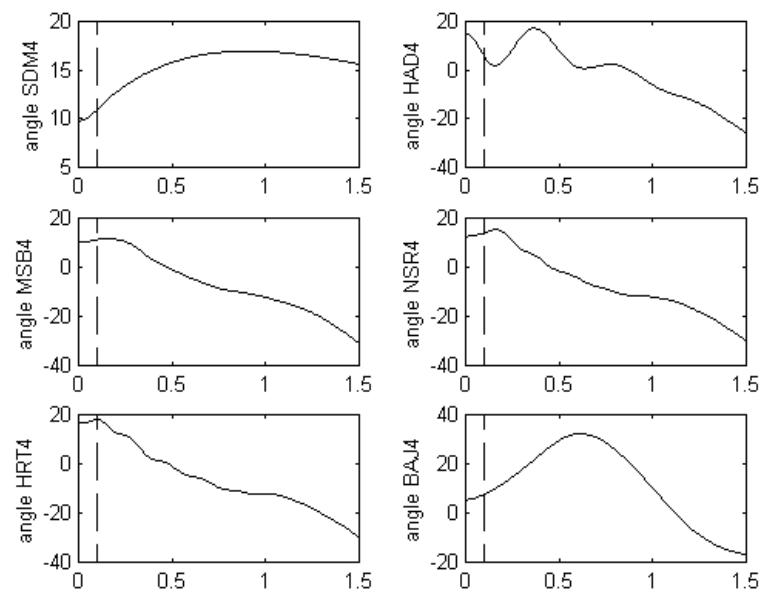

Fig. 8: $\delta$ vs. $\mathrm{t}$ for all generating units in situation of BAJ4 outage applying the new structure
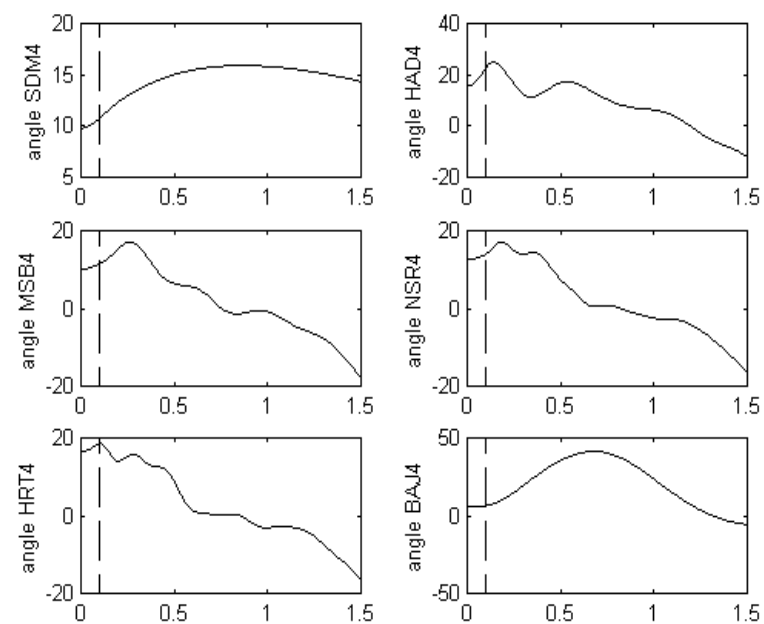

Fig. 9: $\delta$ vs. $t$ for all generating units in situation of HAD4 outages after applying the new structure

represent the load angle against time of all the generating bus bars for these two cases before applying the new structure, while Fig. 8 and 9 shows the same curves after considering the new multi-agent structure.

\section{CONCLUSION}

The aim of this research is improving the performance of the Iraqi Super Grid (400 KV) by using the properties of multi-agent technology. The instability of the power system after a three phase fault at bus bar was predicted using the prediction agent and by applying the control agent the stability of the system is established. The stability behavior of the system has been studied before and after initiation of the control 
agent. The prediction agent initiated the control agent automatically if the prediction process shows that the system is unstable. It seems obvious that this new program greatly improves the ability to monitor network stability, automated computation and speed up the decision making process of the operator.

\section{REFERENCES}

1. Liu, C.C., J. Jung, G.T. Heydt, V. Vittal and A.G. Phadke, 2000. The strategic power infrastructure defense (SPID) system. IEEE Control Syst. Mag., 20: 40-52.

2. Kumar, Y. and A. Shandilya, 1998. Development of Algorithm for Ranking of Contingency and System Stability. IEEE Power Quality Conference, pp: 123-128.

3. Karady, G., A. Daoud and M. Mohamed, 2002. On-line transient stability enhancement using multi-agent technique. IEEE Power Engineering Society Winter Meeting Conference, pp: 893-899.
4. Mansour A. Mohamed, George G. Karady and Ali M. Yousef, 2004. new strategy agents to improve power system transient stability. Trans. Eng. Comput. Technol., 3: 1305-5313.

5. Shant K. Avakian, Afaneen Anwar and Ahmed N. Abdalla, 2006. The application of agent technology on the power flow of iraqi supper grid network. Am. J. Applied Sci., 3 (12): 2174-2177.

6. Fu Shuti, Hu Jiaxiao, Ni Yixin, Chen Jianlin and Xu Youfang, 1993. A practical on-line transient stability program. 2nd International Conference on Advance in Power System Control, Operation and Management, Vol. 2.

7. Afaneen Anwar, 2005. Implementation of GIS in transient stability assessment. Ph.D. Thesis, University of Technology, Baghdad, Iraq.

8. National Dispach Center (NDC), 2004. Baghdada, Iraq. 